Keywords: Chronic schizophrenia; Positive symptoms; Inhibitory control; Attentional deficit.

\title{
Attention in patients with chronic schizophrenia: Deficit in inhibitory control and positive symptoms
}

\author{
Flavia S. Galaverna $a^{\star, \star *, \star \star *}$ \\ Carlos A. Morra ${ }^{\star \star \star}$ \\ Adrián M. Bueno ${ }^{\star, * *}$ \\ * Laboratorio de Psicología, Facultad de \\ Psicología, Universidad Nacional \\ de Córdoba, Córdoba \\ ** Carrera de Psicología, Facultad de \\ Filosofía y Humanidades, Universidad \\ Católica de Córdoba, Córdoba \\ *** Sanatorio "Profesor León Morra", Córdoba \\ ARGENTINA
}

\begin{abstract}
Background and Objectives: Attention is a central mechanism controlling information processing, activating and inhibiting processes, and forming a complex system including diferent networks in specific areas of the brain ${ }^{1}$. To correctly assess the role of attention in schizophrenia it is necessary to discriminate its different attentional components, which may by selectively altered. Attention span, focused attention, selective attention, sustained attention and inhibitory response, were assessed in patients with chronic schizophrenia and healthy matched controls.

Methods: The study included 32 patients diagnosed with chronic schizophrenia and 32 healthy subjects. The groups were matched in age, sex, and level of education. Symptom severity (positive symptoms, negative symptoms, and general psychopathology) was assessed with the Scale for the Assessment of Positive and Negative Symptoms (SAPS and SANS). Attentional components were measured by Forward Digit Span, Symbol Search, Digit Symbol Coding, Stroop Test and Picture Completion.

Results: Schizophrenic patients exhibited lower attentional scores in all tests compared to the control group. Inhibitory control and sustained attention were the most affected traits in schizophrenic patients. An inverse correlation was observed between inhibitory control and delusions and disorganized thinking. No significant correlations were observed between negative symptoms and attentional performance.

Conclusions: The pattern of results obtained in this paper evidences the role of an inhibitory control deficit in patients with chronic schizophrenia that could also be involved in other attentional and cognitive failures, and also be connected to positive symptoms.
\end{abstract}

Received: 8 September 2011

Revised: 1 April 2012

Accepted: 29 May 2012 


\section{Introduction}

Attention deficits are a main neurocognitive feature of schizophrenia. These disorders have been observed in children with high genetic risk of schizophrenia and in the direct relatives of patients ${ }^{2-4}$. Moreover, attention seems to be the neurocognitive predictor of motor skill ability and social problem solving 5 .

Given the conceptual, neuroanatomical and neurofunctional complexity of attention, it cannot be reduced to a simple definition, linked to a single anatomic structure, or assessed by a single test ${ }^{6}$. Attention is a central mechanism for the control of information processing, activating and inhibiting processes, and constituting a complex system that involves different networks in specific areas of the brain ${ }^{1}$.

Attention is constituted by several subsystems, each of which is responsible for different operations including selection or focalization, sustained attention and inhibitory attentional control. It is important to discriminate these different components of attention to correctly assess its role in schizophrenia, as they may be altered selectively.

The components most studied in schizophrenia are selective attention and sustained attention. Posner ${ }^{7}$ established that selective attention involves performance when there are conflicts between signals. Attention has the role of selecting some signals for higher levels of processing while preventing other signals to access those same high levels of processing. Studies on the function of this component in schizophrenia are still controversial. Some authors have found that selective attention is intact for visual tasks 8,9 , while others show that it has a specific role in the inability to correctly perceive reality in schizophrenia ${ }^{10}$.
On the contrary, there is consistent evidence regarding alterations in sustained attention. Sustained attention is the ability to maintain attention and remain in a state of vigilance during a determined period of time $^{11}$. Some studies suggest that sustained attention is a valuable endophenotype for schizophrenia $^{12}$. However recently, Carter et $a l .{ }^{13}$ argued that the attention deficits of patients with schizophrenia probably reflect a deficit in the modulation of the brain activity in response to transitory variations instead of a deficit in sustained attention.

Failures in both these components of attention, selective and sustained attention, are based on the incapacity of eliminating irrelevant stimuli, which in turn is associated with inhibitory control. Different studies showed that inhibitory control is affected in schizophrenic patients ${ }^{14-17}$. This component has been related to the correct function of the executive attention system that participates in tasks involving conflicts within systems, where it is necessary to inhibit usual responses $^{18}$.

These deficits in attention have been related to psychotic symptoms and this association is widely discussed. Some authors have found an inverse relation between the severity of negative symptomatology and the performance of schizophrenic patients in attention tasks, with no significant relation to positive symptomatology ${ }^{19}$. On the contrary, other authors, like Krabbendam et al. ${ }^{20}$, have observed an inverse relation between positive symptoms, especially disorganization, and failures in inhibitory attentional control.

Based on this contradictory background regarding the importance of the different components of attention in schizophrenic patients, the object of the present study was to assess and compare the role of attention span, selective attention, sustained attention and 
inhibitory attentional control processing in chronic schizophrenia. Our main hypothesis was that patients with chronic schizophrenia show general attention alterations, with the inhibitory component being the most affected. Additionally, any connections observed between the function of each attentional component and positive ad negative schizophrenic symptomatology were also studied.

\section{Materials and methods}

\section{Subjects}

The present study included 64 individuals; 32 hospitalized patients diagnosed with chronic schizophrenia (Schizophrenic Group), and 32 healthy matched individuals (ages 30 to 65 of both sexes) (Control Group). All patients were hospitalized at the Sanatorio Profesor León Morra and the Clínica San Nicolás. The patients had to be psychiatrically stable for at least two weeks on their treatment with antipsychotic drugs before participating in the study. Healthy participants were included considering the following criteria: a) they had no history of any neurological or psychiatry disorders or head injuries; b) they had no history of substance abuse/dependence. All participants were clearly informed regarding the aim of the study and assessment manipulations, and gave their written informed consent prior the beginning of the study.

\section{Measures}

Symptom assessment. A Spanish version of the Scale for the Assessment of Positive Symptoms (SAPS) ${ }^{21}$ and the Scale for the Assessment of Negative Symptoms (SANS) ${ }^{22}$ were used for assessing the severity of the clinical syndrome.
Neuropsychological Tasks. The tests have been chosen to investigate the attentional components involved in schizophrenia are multicomponent, being that each of these involves the coordinated participation of several functions. For example, in most tests used here in addition to assessing attentional function, also assess processing speed (Stroop Tests, Symbol Search, Digit Symbol). However, try to isolate certain components as reported in the literature.

- Digit Span Forward ${ }^{23}$. In this task, the participant has to repeat a sequence of numbers exactly as the examiner reads them. If correctly repeated, the examiner reads the next set of numbers, and continues until the participant fails in two series. The amount of digits increases in each subsequent sequence. The test score is the highest number of digits repeated correctly. The forward digit span was used to measure the effectiveness of attention, especially the attention $\operatorname{span}^{24,25}$.

- Symbol Search ${ }^{23}$. The participant is presented with a series of paired groups, each consisting of a target group and search group. The participant must decide whether either of the target symbols is in the search group, made up of five search symbols. The total score is the number of correct answers minus the number of incorrect answers. This test was used for assessing sustained attention $^{26}$.

- Stroop Color-Word Test ${ }^{27}$. This task has three parts. First, the participant must read outloud the names of the colors 'red', 'green' and 'blue', printed in black, during 45 seconds. Secondly, the participant is shown rows of ' $\mathrm{X}$ ' printed in different colors, and he must name the the colors of each row of ' $x$ ' for 45 seconds. Thirdly, the words "red", "green" and "blue" are printed in dif- 
ferent colors, and the participant must name the colour of the ink the word is printed in, ignoring its meaning, during another 45 seconds. The total score is the number of correct answers in each part. This test was used to evaluate different attentional processes, particularly sustained attention can be evaluated with the first section, and focused attention and inhibitory attentional control with the third ${ }^{24,25}$.

- Digit Symbol-Coding ${ }^{23}$. This task consists of rows containing small blank squares, each paired with a randomly assigned number from one to nine. A key is printed above these rows pairing each number with a different nonsense symbol. Following a practice trial on the first seven squares, the participant must fill in the black spaces with the symbol that is paired to the number above the blank space during $120 \mathrm{sec}-$ onds. In this study, it was especially used to measure sustained attention ${ }^{28}$.

- Picture Completion ${ }^{23}$. The participant is presented with a series of slides with images that have an important part missing. The participant must read and indicate the missing part of the image. The complexity of the test increases as the participant provides a correct answer. The score of the test is the number of correct answers. This test was used to study selective attention ${ }^{24}$.

\section{Statistical Analyses}

Differences in performance between patients and controls were examined using an independent $t$ test with attention. Results were considered significant with $\mathrm{p}<0.05$. Relations between attentional measures and clinical characteristics were investigated using exploratory Pearson's product moment correlations. Correlations between dif- ferent attentional tests in a group of patients were analyzed using the Pearson correlation coefficient. Results were considered significant at $\mathrm{p}<0.05$. All statistical tests were carried out using the PAWS statistics 18 .

\section{Results}

\section{Demographic and clinical results}

Participants were matched in terms of age, gender and educational level achieved. The entire sample was composed by $68.75 \%$ of women and $31.25 \%$ of men, ranging 30 to 65 years old (a mean of 45.44 years old for the control group and 44.87 years old for the patient group). Table 1 shows a detail of the demographic traits.

Regarding the clinical status of the patient group, $58 \%$ of the patients received a diagnosis of paranoid schizophrenia, whereas the remaining $42 \%$ exhibited a non paranoid diagnosis including disorganized and residual subtypes. Patients with catatonic and undifferentiated schizophrenia were not included in the study. The first episode of a psychotic break occurred around 25 years old. The average years of illness was 20 years $(\mathrm{M}=20.00, \mathrm{SD}=2.69)$, which correlates with the chronicity of the patient sample. The vast majority of patients, $84.37 \%$, were medicated with atypical antipsychotics at the time of evaluation, whereas the remaining $15.63 \%$ were medicated with typical antipsychotics.

Patients had similar values for both positive and negative symptoms in the SAPS and SANS scales. In the analysis of specific symptoms for the positive scale, we found that hallucinations were generally rated as moderate, with a frequency of at least once a week. Most hallucinations were experienced as voices commenting and talking, although there were some cases of patients with visu- 
al and kinesthetic hallucinations. Delusions were rated as marked, which are consistently and strongly supported by interfering behavior. Behavioral alterations included mild, occasionally observed unusual behaviors. Formal thought disorder was rated as moderate, with derailment, tangential, incoherent and circumstantial speech and distractibility frequently observed in patients.

Regarding negative symptoms, affective poverty was generally moderate, with reduced facial expressions, spontaneous movements and expressive gestures, poor eye contact and incongruent affective responses. Alogia was rated as mild, with poverty of speech and occasional crashes, and increased response latency. The symptoms of lethargy and apathy were markedly noticeable in the patient group, with a recurring problem in toilet and hygiene, persistence in physical activities and anergy. Anhedonia and antisocial behaviour were both equally valued as high. Attention problems were rated as moderate. A summary of these results is shown in Table 1 .

Table 1

Demographic and clinical characteristics of the schizophrenia patients and healthy comparison subjects

\begin{tabular}{|c|c|c|}
\hline Variable & $\begin{array}{l}\text { Healthy controls } \\
\text { (N 32) }\end{array}$ & $\begin{array}{l}\text { Patients with schizophrenia } \\
\text { (N 32) }\end{array}$ \\
\hline & Mean (SD) / \% & Mean (SD) / \% \\
\hline Age & $45.44(8.55)$ & $44.87(7.83)$ \\
\hline Education (years) & $12.53(3.96)$ & $11.17(3.93)$ \\
\hline $\operatorname{Sex}(\mathrm{F} / \mathrm{M})$ & $68.75 \% / 31.25 \%$ & $68.75 \% / 31.25 \%$ \\
\hline Handedness (R/L) & $93.75 \% / 6.25 \%$ & $90.63 \% / 9.37 \%$ \\
\hline \multicolumn{3}{|l|}{ Subtype } \\
\hline Paranoid/ Non paranoid & - & $58.10 \% / 41.90 \%$ \\
\hline Years of illness & - & $20.00(2.69)$ \\
\hline \multicolumn{3}{|l|}{ Antipsychotic medications } \\
\hline Typical /Atypical & - & $15.63 \% / 84.37 \%$ \\
\hline SAPS $^{\mathrm{a}}$ & - & 58.83 \\
\hline $\mathrm{SANS}^{\mathrm{b}}$ & - & 59.23 \\
\hline Hallucination & - & Moderate \\
\hline Delusions & - & Marked \\
\hline Bizarre behavior & - & Mild \\
\hline Formal thought disorder & - & Moderate \\
\hline Affective flattening or blunting & - & Moderate \\
\hline Alogia & - & Mild \\
\hline Avolition / apathy & - & Marked \\
\hline Anhedonia / asociality & - & Marked \\
\hline Attention & - & Moderate \\
\hline
\end{tabular}

a Scale for the Assessment of Positive Symptoms.

b Scale for the Assessment of Negative Symptoms. 


\section{Attentional performance}

Table 2 shows the descriptive statistics of all the attentional tasks. The $t$ test results (Table 2) comparing the patient group and the healthy control group for different attentional tests were significant. The group of patients with chronic schizophrenia performed significantly lower than expected compared to the healthy control group in all tasks. In the test of attentional span (Digits Forward) the control group achieved a length attentional of 7 digits approximately, while the group of patients achieved 5 digits $(t=6.33 ; p=<0.001)$. In the sustained attention tasks also showed deficits in the performance of the group of patients. For example, in the Symbol Search test the control group averaged 25 correct answers in the two minutes it takes the task, while the group of patients averaged only 10 correct answers $(t=10.29 ; p=<0.001)$. In the task of Digit Symbol Coding, the patient group only achieved complete 27 symbols correctly, while the control group reached 60 symbols $(t=12.55 ; p=<0.001)$. Another case is the Word Stroop task, where patients achieved reading in a loud voice for $45 \mathrm{sec}-$ onds a total of 63 words compared to healthy control group who read a total of 99 words $(t=8.33 ; p=<0.001)$. In the Picture
Completion task of selective attention, the healthy control group was able to establish the missing part of 18 figures, while the schizophrenia group could only do it for 9 figures $(t=8.66 ; p=<0.001)$. Meanwhile, in the Word Colour Stroop task, patients were able to correctly answer only 15 items, compared to healthy control group who managed to correctly say the colour of the ink in which 42 words were printed $(t=$ $11.31 ; p=<0.001)$.

No correlation was observed between attentional performance and years of illness or medication. A significant negative correlation was observed between the assessment of positive symptoms the Stroop Word-Color test $(r=-0.40, p=0.028)$; the presence of delusions was strongly correlated with the performance on this task $(r=-0.42, p=0.01)$. Formal thought disorder was also negatively correlated with this test $(r=-0.40, p=0.02)$.

The assessment of negative symptoms was not significantly correlated with any of the tests. However, the specific analysis of symptoms showed that alogia is negatively correlated with performance in the Digit Span Forward trial $(r=-0.45, \mathrm{p}=0.01)$. The assessment of attention in the SANS was only negatively correlated with the Stroop Word - Color test $(r=-0.37, \mathrm{p}=0.03)$.

Table 2

Mean (SD) scores in performance attentional tasks for schizophrenia patients $(\mathrm{N}=32)$ and healthy comparison subjects $(\mathrm{N}=32)$ and Analysis of Variance results for attentional measure

\begin{tabular}{lccrc} 
Attentional test & $\begin{array}{c}\text { Healthy controls } \\
(\mathrm{N}=32)\end{array}$ & $\begin{array}{c}\text { Patients with schizophrenia } \\
(\mathrm{N}=32)\end{array}$ & Mean (SD) & t test \\
\hline Mean (SD) & $4.77(1.09)$ & 6.339 & $<0.001$ \\
\hline Symbol Search & $6.59(1.18)$ & $9.96(6.90)$ & 10.288 & $<0.001$ \\
Stroop Word & $24.97(5.32)$ & $62.77(22.56)$ & 8.332 & $<0.001$ \\
Stroop Word - Color & $99.24(14.10)$ & $15.73(9.75)$ & 11.314 & $<0.001$ \\
Digit Symbol - Coding & $62.26(11.40)$ & $26.65(10.97)$ & 12.555 & $<0.001$ \\
Picture Completion & $18.26(4.12)$ & $9.19(5.02)$ & 8.663 & $<0.001$ \\
\hline
\end{tabular}




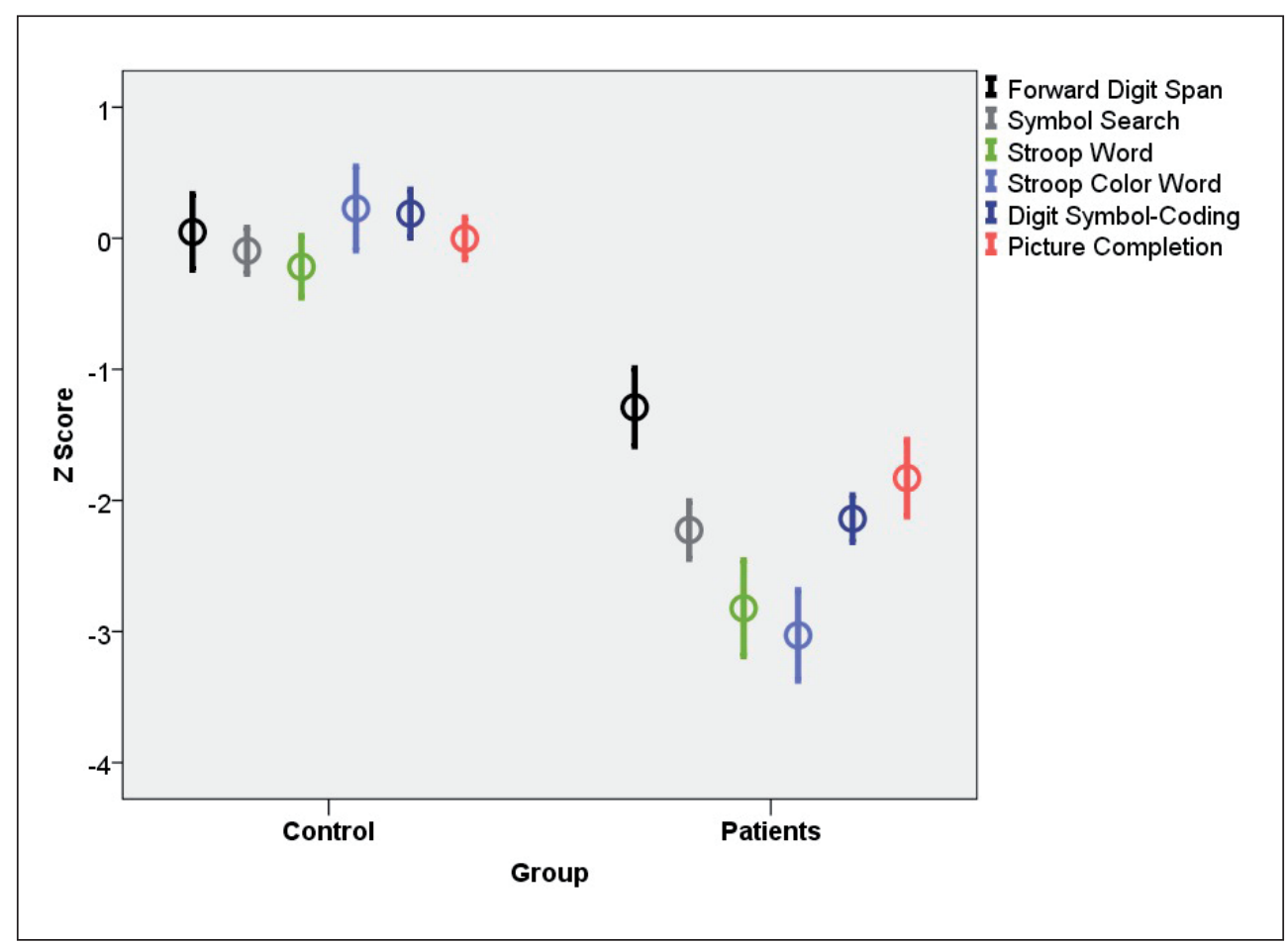

Figure 1. Differences between the control and patient groups in the performance of attentional tasks. $\mathrm{Z}$ scores. The dots indicate the mean and the length of the bars indicate the standard deviation.

\section{Discussion}

The results of the present study confirm the hypothesis that patients with chronic schizophrenia have a general attention deficiency. These results are in accordance with consistent evidence of attention disorders in this clinical population ${ }^{29-32}$. The importance of these results is based on the fact that attention is a central cognitive function enabling precision, rapidity and continuity to information processing ${ }^{33}$, and interacts with other cognitive functions such as sensory perception, memory, language, executive functions, among others. This could explain the existence of generalized failures in different cognitive functions.
The present study specifically focused on the function of different components of attention and found that inhibitory attentional control and sustained attention were the most affected in patients with chronic schizophrenia. These observations are partly supported by other studies that found significant alterations in both these components of attention ${ }^{14,29,34-36}$. Breton et al. ${ }^{14}$, recently found that patients had a specific attention deficient and a significantly worse performance in tests involving the executive attention network, while the alertness and orientating attentional networks remained intact.

Failures in both these components, sustained attention and inhibitory control, could be considered closely related because 
sustained attention works by two mechanisms: one involving excitatory processes, associated with the stimulating object, and the other involving inhibitory mechanisms, associated to unknown stimuli ${ }^{37}$.

In this study we found no significant relations between the global severity of the negative symptoms and the different attentional components. These results are inconsistent with studies that suggest a relationship between severity of negative symptoms and inhibitory control failures. In our study only found that the severity of alogia was associated with short attention span, and problems of attention during the interviews were significantly associated with a deficiency in inhibitory control.

The most significant results were found in relation to the global severity of positive symptoms. Significant inverse correlations were observed between positive symptoms and performance in the Stroop Colour-Word test. Additionally, these results are supported by recent findings that evidence a connection between failures in inhibitory control and the presence of positive symptoms, especially symptoms of disorganization ${ }^{14,20}$.

It has been shown that the deficiency in the executive attention network of schizophrenia patients is related to an abnormal function in tasks involving executive processes and some of the characteristic symptoms of the disorder such as formal thought disorder and weak associations ${ }^{14}$. The problems patients have in the Stroop ColourWord test could be associated with an attenuated activation in the cingulate cortex and left inferior frontal gyrus, as changes in the positive symptoms appear to be correlated with the activation of these areas ${ }^{20}$.
Failures in inhibitory attentional control were found to be related to the presence of delirium and disorganized thinking, and this has not been previously established in other studies. This relation could be explained by regarding attentional control as the ability to guide thought and actions according to changes in the physical context and internal intentions and goals; this ability is the basis for the correct function of other more complex cognitive processes ${ }^{35}$.

In favour of these results, there are references of studies in patients with methamphetamine abuse that establish a possible relation between the frequency of paranoid delirium and the magnitude of Stroop interference $^{39}$. Thus, it is possible to hypothesize that alterations in the dopaminergic function (well-established in schizophrenia and in methamphetamine abuse) could be associated to attention abnormalities, especially inhibitory control and the appearance of delirium symptoms ${ }^{39}$.

The pattern of results of this study suggests the presence of a deficient inhibitory control in patients with chronic schizophrenia, which could also have an important role in other attention and cognitive failures, as well as in positive symptomatology. Future investigations should further analyze the relations between sustained attention, inhibitory control, and the schizophrenic symptoms that are closely linked with thought and language. Moreover, and considering the fact that inhibitory control has been included in the executive attention system ${ }^{18}$, it would also be interesting to study the relation between attention and executive functions, such as working memory and cognitive flexibility, which have been associated with attentional control ${ }^{40}$. 


\section{Role of the funding source}

FSG is a beneficiary of a doctoral research fellowship PDFT SECYT-UNC FONCYT, from the Secretary of Science and Technology of the UNC, in the framework of the Human Resources Training ANPCyT. The SECYT-UNC FONCYT has had no further role in study design, collection, analysis and interpretation of data, writing of the report, or the decision to submit the paper for publication.

\section{Contributors}

FSG participated in the literature review, data extraction and first draft of the manuscript draft. AMB and CAM participated in the conception of the study and provided scientific supervision. IRO and MJK provided statistical advice and scientific supervision. MCFF, ASF and DAP helped draft the manuscript. FG contributed data extraction, literature review and contribuited with the written manuscript. FSG, AMB and CAM contributed content knowledge and made suggestions on the written manuscript. All authors read and approved the final manuscript.

\section{Acknowledgements}

We thank to Sabrina Trovatto, Florencia Di Yorio and Fernando Gay, for their kind, generous help while testing patients.

\section{References}

1. Posner MI, Raichle ME. Images of mind. New York: Scientific American Library; 1997.

2. Erlenmeyer-Kimling L, Cornblatt BA. A summary of attentional findings in the New York High-Risk Project. J Psychiatr Res 1992; 26: 405-426.

3. Filbey FM, Toulopoulou T, Morris RG, McDonald C, Bramon E, Walshe M, et al. Selective attention deficits reflect increased genetic vulnerability to schizophrenia. Schizophr Res 2008; 101(1): 169-175.

4. Rutschmann J, Cornblatt B, Erlenmeyer-Kimling L. Sustained attention in children at risk for schizophrenia: findings with two visual continuous performance tests in a new sample. J Abnorm Child Psychol 1986; 14: 365-385.

5. Green MF, Kern RS, Braff DL, Mintz J. Neurocognitive deficits and functional outcome in schizophrenia: Are we measuring the "right stuff"? Schizophr Bull 2000; 26 (1): 119-136

6. Zomeren AH, Brouwer WH. Theories and concepts of attention. In: Zomeren AH, Brouwer WH, editors. Clinical neuropsychology of attention. New York: Oxford University Press; 1994. p. 7-36.

7. Posner MI. Structures and functions of selective attention. In: Boll T, Bryant B, editors. Master Lectures in Clinical Neuropsychology and Brain Function: Research, Measurement, and Practice. American Psychological Association; 1987. p. 171-202.

8. Elahipanah A, Christensen BK, Reingold EM. Visual selective attention among persons with schizophrenia: The distractor ratio effect. Schizophr Res 2008; 105: 61-67.

9. Gold JM, Fuller RL, Robinson BM, McMahon RP, Braun EL, Luck SJ. Intact Attentional Control of Working Memory Encoding in Schizophrenia. J Abnorm Psychol 2006; 115: 658-673.

10. Brébion G, Smith MJ, Gorman JM, Amador X. Reality monitoring failure in schizophrenia: The role of selective attention. Schizophr Res 1996; 22 (2): 173-180.

11. Parasuraman R. Sustained attention in detection and discrimination. In: Parasuraman R, Davies DR, editors. Varietes of attention. San Diego, CA: Academic Press; 1984. p. 243-271.

12. Lennertz L, Wagner M, Frommann I, SchulzeRauschenbach S, Schuhmacher A, Kühn KU, et al. A coding variant of the novel serotonin receptor subunit 5-HT3E influences sustained attention in schizophrenia patients. Eur Neuropsychopharmacol 2010; 20 (6): 414-420. 
13. Carter JD, Bizzell J, Kim C, Bellion C, Carpenter KLH, Dichter G, et al. Attention deficits in schizophrenia Preliminary evidence of dissociable transient and sustained deficits. Schizophr Res 2010; 122 (1): 104-112.

14. Breton F, Planté A, Legauffre C, Morel N, Adès J, Gorwood P, et al. The executive control of attention differentiates patients with schizophrenia, their first-degree relatives and healthy controls. Neuropsychologia 2011; 49 (2): 203-208.

15. Fuller RL, Luck SJ, Braun EL, Robinson BM, McMahon RP, Gold JM. Impaired Control of Visual Attention in Schizophrenia. J Abnorm Psychol 2006; 115 (2): 266-275.

16. Gooding JG, Braun, Studer JA. Attentional network task performance in patients with schizophrenia-spectrum disorders: Evidence of a specific deficit. Schizophr Res 2006; 88: 169-178.

17. Wang K, Fan J, Dong Y, Wang CQ, Lee TM, Posner MI. Selective impairment of attentional networks of orienting and executive control in schizophrenia. Schizophr Res 2005; 78: 235-241.

18. Posner MI, Fernandez Duque D. Attention in the human brain. In: Wilson RA, Keil FC, editors. The MIT encyclopedia of the cognitive sciences. Cambridge, MA: MIT Press; 1999.

19. O Gráda C, Barry S, McGlade N, Behan C, Haq F, Hayden J, et al. Does the ability to sustain attention underlie symptom severity in schizophrenia? Schizophr Res 2009; 107: 319-323.

20. Krabbendam L, O’Daly O, Morley LA, van Os J, Murray RM, Shergill SS. Using the Stroop task to investigate the neural correlates of symptom change in schizophrenia. Br J Psychiatry 2009; 194: 373-374.

21. Andreasen NC. Scale for the Assessment of Negative Symptoms (SANS). Iowa City, IA: University of Iowa; 1984.

22. Andreasen NC. Scale for the Assessment of Positive Symptoms (SAPS). Iowa City, IA: University of Iowa; 1984.

23. Weschler D. Wechsler Adult Intelligence Scale. Administration and Scoring Manual, third ed. Buenos Aires, Argentina: Paidós; 2006.

24. Cohen RA, Salloway S, Sweet LH. Neuropsychiatric Aspects of Disorders of Attention. In: Yudofsky SC, Hales RE, editors. The American Psychiatric Publishing textbook of neuropsychiatry and behavioral neurosciences, $5^{\text {th }}$ ed. Washington, DC: American Psychiatric Publishing; 2008. p. 405-444.
25. Lezak MD, Howieson DB, Loring DW. Orientation and attention. In: Lezak md, Howieson DB, Loring DW. Neuropsychological assessment, $4^{\text {th }}$ ed. New York: Oxford University Press; 2004. p. 337-374.

26. Mesulam MM. Attention, confusional state, and attention. In: Mesulam MM, editor. Principles of Behavioral Neurology. Philadelphia: FA Davis; 1985. p. 125-168.

27. Golden CJ. Stroop Color and Word Test: Manual for clinical and experimental uses. Chicago: Stoeling; 1978.

28. Buyske S, Bates ME, Gharani N, Matise TC, Tischfield JA, Manowitz P. Cognitive Traits Link to Human Chromosomal Regions. Behav Genet 2006; 36(1): 65-76.

29. Filbey FM, Russell T, Morris RG, Murray RM, McDonald C. Functional magnetic resonance imaging (fMRI) of attention processes in presumed obligate carriers of schizophrenia: preliminary findings. Ann Gen Psychiatry 2008; 7: 18 .

30. Kurtz MM, Moberg PJ, Gur RC, Gur RE. Approaches to Cognitive Remediation of Neuropsychological Deficits in Schizophrenia: A Review and Meta-Analysis. Neuropsychol Rev 2001; 11: 197-210.

31. Nieuwenstein MR, Aleman A, de Haan EH. Relationship between symptoms dimensions and neurocognitive functioning in schizophrenia: a meta-analysis of WCST and CPT studies. J Psychiatr Res 2001; 35: 119-125.

32. Luck SJ, Gold JM. The Construct of Attention in Schizophrenia. Biological Psychiatry 2008; 64 (1): 34-39.

33. LaBerge D. Attentional Processing: The Brain's Art of Mindfulness. Cambridge, MA: Harvard University Press; 1995.

34. Bozikas VP, Andreou C, Giannakou M, Tonia T, Anezoulaki D, Karavatos A, et al. Deficits in sustained attention in schizophrenia but not in bipolar disorder. Schizophr Res 2005; 78: 225-233.

35. Chen WJ, Faraone SV. Sustained attention deficits as markers of genetic susceptibility to schizophrenia. Am J Med Genet 2000; 97 (1): 52-57.

36. Harris JG, Minassian A, Perry W. Stability of attention deficits in schizophrenia. Schizophr Res 2007; 91: 107-111.

37. Neill WT, Westberry RL. Selective attention and the suppression of cognitive noise. J Exp Psychol Learn Mem Cogn 1987; 13: 327-334

38. Cohen JD, Botvinick M, Carter CS. Anterior cingulate and prefrontal cortex: Who's in control? Nat Neurosci 2000; 3: 421-423. 
39. Salo R, Nordahl TE, Possin K, Leamon M, Gibson DR, Galloway GP, et al. Preliminary evidence of reduced cognitive inhibition in methamphetamine-dependent individuals. Psychiatry Res 2002; 111: 65-74.

40. Ríos Lago M, Periáñez JA, Rodríguez JM. Neuropsicología de la atención. In: Tirapu Ustárroz J, Ríos Lago M, Maestú Unturbe F, editors. Manual de Neuropsicología. Barcelona: Viguera Press; 2008; p. 149-188.
Author for correspondence:

Flavia S. Galaverna

Laboratorio de Psicología, Facultad de Psicología Universidad Nacional de Córdoba

Enfermera Gordillo esquina Enrique Barros

Ciudad Universitaria, CP X5000GYA

Córdoba, Argentina

Tel.: + 543514344984

Fax: + 543514334064

E-mail: fgalaverna@psyche.unc.edu.ar 